\title{
Application of Synthetic Peptides to Improve Parameters of Skin Physiology: An Open Observational 30-Day Study
}

\author{
Neil Fisher $^{1^{*}(\mathbb{D})}$, Davide Carati \\ ${ }^{1}$ Contrad Swiss SA, Lugano, Switzerland \\ ${ }^{2}$ Ekuberg Pharma s.r.l., Martano, Italy \\ Email: ${ }^{\star}$ neil.fisher@contrad.ch
}

How to cite this paper: Fisher, N. and Carati, D. (2020) Application of Synthetic Peptides to Improve Parameters of Skin Physiology: An Open Observational 30-Day Study. Journal of Cosmetics, Dermatological Sciences and Applications, 10, 163-175. https://doi.org/10.4236/jcdsa.2020.104018

Received: September 3, 2020

Accepted: October 23, 2020

Published: October 26, 2020

Copyright $\odot 2020$ by author(s) and Scientific Research Publishing Inc. This work is licensed under the Creative Commons Attribution International License (CC BY 4.0).

http://creativecommons.org/licenses/by/4.0/

\begin{abstract}
We describe a novel, non-invasive peptide-based product intended to counter several pathophysiological signs of aged skin through the topical application of signalling molecule mimics. Several aesthetic parameters of skin physiology were assessed. The product was tested on 20 healthy Caucasian women (35 - 55 years-old) who applied the product twice-daily to the face for 30 days. Skin elasticity, firmness, sagging, brightness, and luminosity were assessed. Additionally, over a period of 3 hours, the hydrating effect of the product vs placebo vs non-treated skin was assessed. After 30 days, participants showed significant increases in skin elasticity $(11.8 \%$ increase, $\mathrm{p}<0.01)$, luminosity (2.5\% increase, $\mathrm{p}<0.01)$, and brightness $(110 \%$ increase, $\mathrm{p}<0.001)$ with concomitant significant decreases in skin sagging $(6.3 \%$ decrease, $\mathrm{p}<0.05)$. The product gave a rapid and sustained hydrating action that was significantly greater than placebo alone $(10.2 \%$ increase, $\mathrm{p}<0.05)$. No adverse reactions were reported. Cosmetic products based on the action of synthetic peptide mimics of endogenous signalling molecules show promise for combatting the pathological degradation of skin as seen in the elderly. Strategies to reverse these symptoms through non-invasive techniques warrant further investigation. This study provides support for the application of peptides to combat signs of aging and to improve the general condition of the skin.
\end{abstract}

\section{Keywords}

Peptides, Elasticity, Moisture, Skin, Topical

\section{Introduction}

As the skin ages, various physiological processes contribute to the external signs 
of aging. These processes can be divided into either intrinsic or extrinsic factors. Intrinsic factors, such as genetic variation [1] [2] and oxidative stress [3] [4], have been suggested to contribute to approximately $50 \%$ of the skin aging process [1] (although the percentage due to extrinsic factors is much lower in youth and increases with age and the accumulation of extrinsic effects [2]). Extrinsic factors responsible for skin aging include UVB radiation [5] (photo-aging), pollution [6], and cigarette smoking [7]. Aged skin contains fewer collagen type-I fibrils and a greater degree of collagen fibril fragmentation [3] which contributes to reduction in skin mechanical tension (or firmness). Dermal fibroblasts in aged ( $>80$ year-old) skin secrete higher levels of matrix metalloprotease 1 (compared to 21 - 30 year-old skin) [3]. Aged skin is also thinner (both epidermis and dermis), contains fewer blood vessels, lower amounts of collagen, more fragmented collagen, and is less elastic, less resistant to insult and injury and takes longer to heal [8]. Aged skin is therefore not only an aesthetic concern, but also one that has significant implications for public health as greater numbers of people survive into old age.

Peptides which mimic the action of endogenous signalling molecules to stimulate innate cellular repair pathways show promise for skin regeneration though targeted regulation of signalling pathways involving inflammation, oxidation, and enzyme-related degradation of extracellular matrix proteins [9] [10] [11] [12]. Additionally, by stimulating protein production through endogenous pathways, the introduction of incorrectly folded or modified proteins can be minimised, as could be the case with injection of exogenously-produced non-native proteins [13]. The idea of using peptides in cosmetics is not new [14], and interest in their application is growing as evidenced by the increasing number of articles in the literature.

We selected 4 recombinant peptides synthesised to mimic the signalling action of molecules with known effects on skin growth, healing, and homeostasis. SH-Oligopeptide-1, a 53-amino acid recombinant isomer of epidermal growth factor (EGF), which has long been associated with connect tissue repair [15], has been shown to enhance the healing of canine oral soft-tissue wound model [16] and has generated promising results in the treatment of a variety of human skin lesions [17]. SH-Oligopeptide-2 codes for an isomer of human insulin-like growth factor (IGF), which has a protective effect against UVB-mediated transformation in keratinocytes [18] [19], as well as positive effects on the healing of skin ulcers [20] and a long-demonstrated positive effect on elasticity [21] [22]. SH-Polypeptide-1 codes for an isomer of basic fibroblast growth factor (FGF-2). FGF-2 has been recently shown to stimulate mitogenesis, angiogenesis and adipogenesis when implanted subcutaneously [23] and to increase neovascularization and assist in scar tissue remodelling after myocardial infarction [24] as well as inhibit inflammatory factors and improve skin wound healing [25]. Finally, SH-Polypeptide-42, synthesised to mimic interleukin 7 (IL-7) which has been shown to be produced and released by dermal endothelial cells [26] and kerati- 
nocytes [27], and to increase keratinocyte migration rates in chronic wounds [28]. It also plays a central role in the survival the development of lymphoid cells through the activation of the phosphatidylinositol 3 kinase (PI3K)/protein kinase B (Akt) signalling pathway [28] [29].

Here, we present a clinical assessment of skin physiology parameters, before and after application of a novel product containing 4 recombinant human peptide isomers at low concentration $(<0.0025 \%)$ configured to mimic the action of endogenous signalling molecules implicated in the control of various aspects of skin biology.

\section{Materials and Methods}

Twenty healthy female Caucasian participants aged between 35 and 55 years of age (median age: 54 years old) were enrolled in a 30-day open observational study during March and April 2019. Participants were enrolled from the study centre (Abich Clinical and Cosmetic Testing Centre, Milan, Italy) database of volunteers after meeting all inclusion criteria and none of the exclusion criteria. Participants were informed of the aims of the study, the procedures and the possible risks involved in the study, and freely gave their informed consent following the principles outlined in the Declaration of Helsinki.

Inclusion criteria included: female gender, aged 35 - 55 years, with visible wrinkles around the eyes. Exclusion criteria included: pregnancy, or breastfeeding, the use of medication that could affect the response of the skin, signs of skin irritation at any of the test sites (periocular area, cheeks and forearm area), participation in other simultaneous studies or recent previous studies without a suitable rest period that might interfere with the test evaluation, judged as not suitable by a medical doctor. During the study the following withdrawal criteria were applied: development of illness or accident or condition which could affect the outcome of the study, intolerance to the tested product, participants that no longer wished to participate in the study. Twenty participants were screened, enrolled and all twenty completed the study; there were no screen failures or withdrawals. For the duration of the study, participants were asked to refrain from using similar products in the analysed areas.

The tested product was a clear odourless hydrogel (The Actuator, Contrad Swiss SA, Switzerland), the composite ingredients of which are reported in Table 1. All participants were instructed on how to apply the product (approximately 150 - $300 \mu \mathrm{l}$ per application) to the entire facial area twice a day for the 30-day study period. All participants were assessed for each of the assessed parameters both before, and after, use of the product. All measurements were performed by trained staff. Data was collected at a certified GLP test facility by trained personnel, under controlled environmental conditions where temperature and humidity were monitored and maintained constant at each measurement time point $\left(20^{\circ} \mathrm{C}+/-2^{\circ} \mathrm{C}, 50 \%+/-5 \%\right.$ relative humidity). There was no formal acclimation time. Tests were not blinded. 
Table 1. Components of the tested product (INCI nomenclature).

Product components

Aqua, Glycerin, Propylene glycol, Carbomer, Sodium hyaluronate, Xanthan gum, Panthenol, PEG-40 hydrogenated castor oil, SH-Oligopeptide-2, SH-Polypeptide-1, SH-Oligopeptide-1, SH-Polypeptide-42, Phenoxyethanol, Dehydroacetic acid, Benzoic acid, Ethylhexylglycerin, Disodium EDTA, Sodium hydroxide.

Assessment of the following skin parameters were performed on the cheek area of both sides of the face before and after 30-days of twice-daily use: elasticity, firmness, sagging. brightness, luminosity. For tests of moisturizing capacity, the tested product was compared to a placebo HA-based hydrogel which consisted of the same hydrogel base as the tested product, but without the 4 peptides; additional comparisons were made to non-treated (NT) skin. Assessment of skin hydration was performed over the course of 3 hours on the inner forearm area; the tested product (approximately $150 \mu \mathrm{l}$ ) was applied to one forearm, the placebo was applied to the same area on the contralateral forearm. Non-treated (NT) measurements were made on the inner upper arm area. All participants received all three treatments (product, placebo, NT).

For the evaluation of skin elasticity, firmness, and sagging a multi-probe adapter (Courage-Khazaka GmbH, Cologne, Germany), equipped with a Cutometer (Courage-Khazaka) was used as described elsewhere [30]. Skin firmness (R0) represents the resistance of the skin to being deformed under negative pressure (450 mbar) and is calculated as the distance that the skin travels up the internal tube of the cutometer (range: $1-10 \mathrm{~mm}$ ). A lower value indicates greater firmness. Elasticity (R2) quantifies the extent to which the skin returns to its original state after deformation. On a scale from 0 to 1 , the closer the value is to 1 , the more elastic is the skin. The sagging (R9) indicates the ability of the skin to return to its original state after multiple cycles of suction and release, the lower the R9 value, the less saggy is the skin. The evaluation of skin firmness, elasticity and sagging was carried on the cheek area of all participants on both sides of the face prior to (T0), and after 30 days (T30d), of twice-daily use of the product. The measuring probe ( $2 \mathrm{~mm}$ diameter) of the Cutometer was positioned perpendicularly to the tested skin area. Suction and release times were both set at 2 seconds, as per the manufacturer's recommendations. Measurements were performed in triplicate.

The evaluation of skin luminosity $\left(\mathrm{L}^{\star}\right)$ was carried out on the cheek area of all participants of both sides of the face at baseline (T0) and after 30 days (T30d) of twice-daily application of the product using a Chromameter CR-200 (Minolta, Tokyo, Japan). The three coordinates of the CIE $1976\left(\mathrm{~L}^{*}, \mathrm{a}^{*}, \mathrm{~b}^{*}\right)$ colour space (also called CIELAB colour space) characterise the sample in terms of its position on three colour scales. $L^{*}=0$ yields black and $L^{*}=100$ indicates diffuse white; negative $\mathrm{a}^{*}$ values indicate green while positive $\mathrm{a}^{*}$ values indicate magenta; and negative $b^{\star}$ values indicate blue while positive $b^{\star}$ values indicate yellow. 
The objective evaluation of the brightness of the face was carried out by specialised staff who analysed and scored digital images of both sides of all 20 participants' faces from T0 and T30d using a 4-point scale (Table 2).

Assessment of skin hydration was performed by using a previously described technique [31]. Briefly, the change in skin electrical capacitance at a depth of approximately $15 \mu \mathrm{m}$ as assessed with a Corneometer CM285 (Courage-Khazaka $\mathrm{GmbH}$ ) on the forearms of participants. Duplicate measurements were taken prior (T0) to one application of either the product, a placebo hydrogel, or no treatment (NT), then after 30 minutes, 60 minutes, and 180 minutes. The placebo was the hydrogel formulation (Table 1) but without peptides. Each measurement was carried out twice per tested area, per participant.

At the end of the study, participants answered a questionnaire regarding the product's pleasantness, texture, and efficacy, as well as their general opinion of the product. Data regarding the tolerability (presence of irritation, itching, burning, redness) of the product was also collected in the questionnaire.

The primary objectives of this study were to assess the tolerability and feasibility of use of a cosmetic product for twice-daily use in 20 women over 30 days. The secondary objectives were to assess the moisturizing, brightening, elasticizing, densifying, and anti-sagging efficacy of the same cosmetic product.

Statistical analyses were carried out using the statistical environment, $\mathrm{R}$ [32]. Tests for normality (Shapiro) dictated use of parametric (paired t-test)/non-parametric tests (Wilcoxon). The effect of the different treatments on hydration data was tested by pairwise t-tests at each time point with Bonferroni correction for multiple comparisons, an analysis of variance (ANOVA, Tukey multiple comparisons of means, $95 \%$ family-wise confidence level) was also performed. The threshold for statistical significance was set at $5 \%(\mathrm{P}<0.05)$. Changes seen over the course of 30 days were calculated as percentage change ((new value - old value) $/($ old value $)) \times 100$ for each individual; the mean of the percentage change was used. Contemporaneous differences between different treatments were calculated as percentage difference ((new value - old value)/(mean of new and old values $)) \times 100$; the mean of the percentage difference was used.

\section{Results}

All 20 participants completed the 30-day study, no adverse reactions or tolerability

Table 2. Skin brightness scale.

\begin{tabular}{cc}
\hline Skin Brightness & Score \\
\hline Not bright & 0 \\
Not very bright & 1 \\
Bright & 2 \\
Very bright & 3 \\
\hline
\end{tabular}


issues were reported. Over the course of 30 days, there were significant changes in most of the assessed parameters. Skin elasticity was increased $11.8 \%$ after 30 days relative to initial values $(P=0.00006$, Wilcoxon test, $n=20)$ (Figure 1$)$. Skin firmness was increased $1.4 \%$ over time with use of the tested product ( $\mathrm{P}=$ 0.47 , paired t-test, $\mathrm{n}=20$ ) (Figure 1 ). Contrary to firmness and elasticity, the sagging (Figure 1 ) was significantly reduced $6.3 \%(P=0.026$, paired $t$-test, $n=$ 20) over the test period.
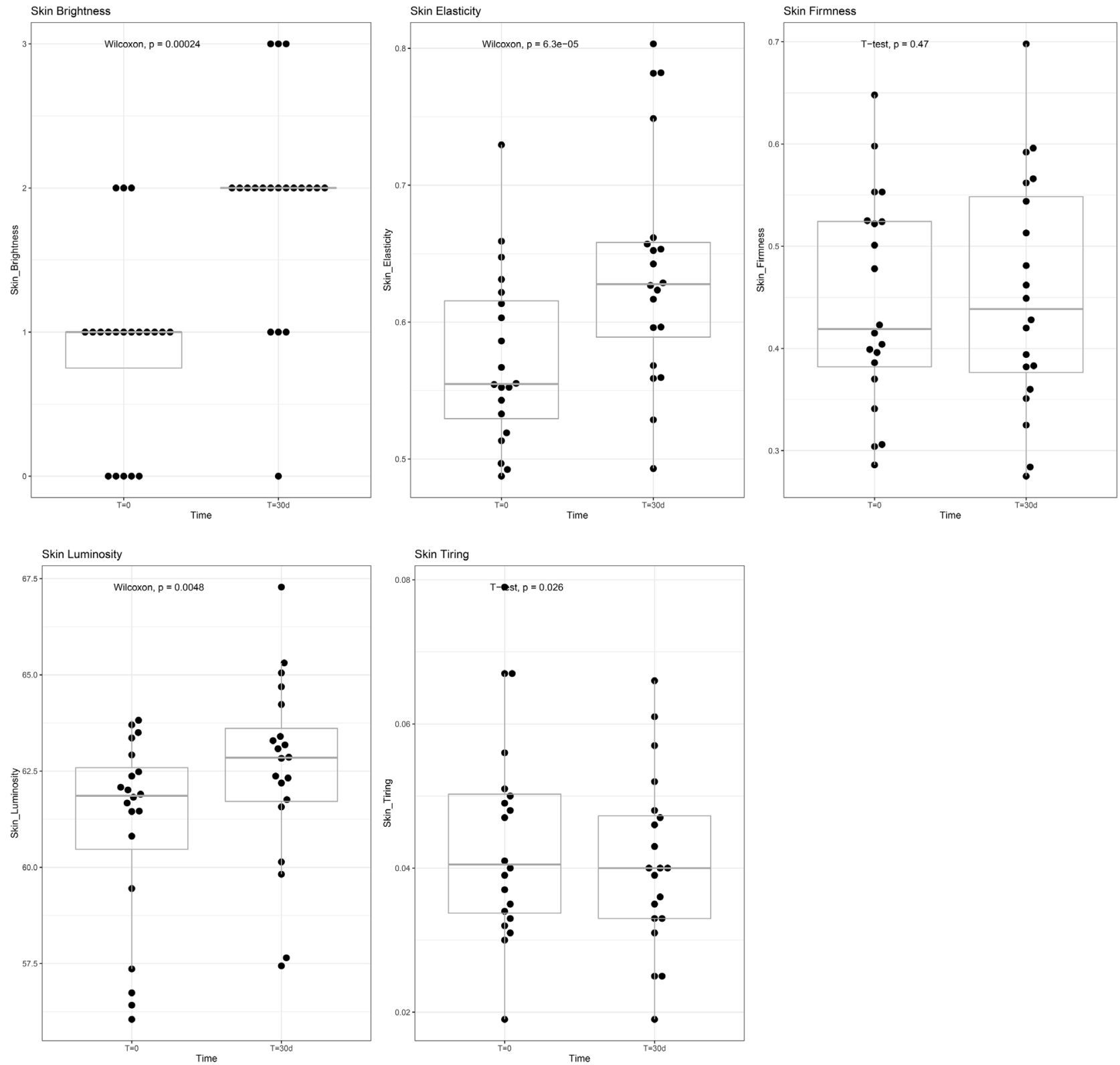

Figure 1. Measurements of skin physiology were assessed prior to, and after, 30 days of twice-daily application of the tested product to the entire facial area. Elasticity, firmness, and sagging of the facial skin was assessed using a Cutometer on both left and right sides of the face. Skin brightness was assessed from photographs of the facial area. A Chromameter was used to assess the luminosity value of the facial skin. In all cases, the mean of repeated measurements on the left and right sides of the face are shown. 
The visual aspect of the skin was assessed by luminosity and brightness. Skin brightness significantly increased ( $\mathrm{P}=0.00024$, Wilcoxon test, $\mathrm{n}=20$ ) with a mean increase in brightness score from 0.9 to 1.9 (range $=0-3$ ) over the course of the study (Figure 1). Increased values were reported for $80 \%(16 / 20)$ participants while 4 participants showed no change in skin brightness. Increases in skin brightness were accompanied by an average increase in luminosity of $2.5 \%(\mathrm{P}=$ 0.0048, Wilcoxon test, $\mathrm{n}=20$ ) over the treatment period from a baseline mean $L^{\star}$ value of 61.07 to 62.52 (Figure 1 ).

Assessment of skin hydration revealed a significant effect of different treatments on hydration (ANOVA, Tukey multiple comparisons of means, $95 \%$ family-wise confidence level, Table 3 and Figure 2).

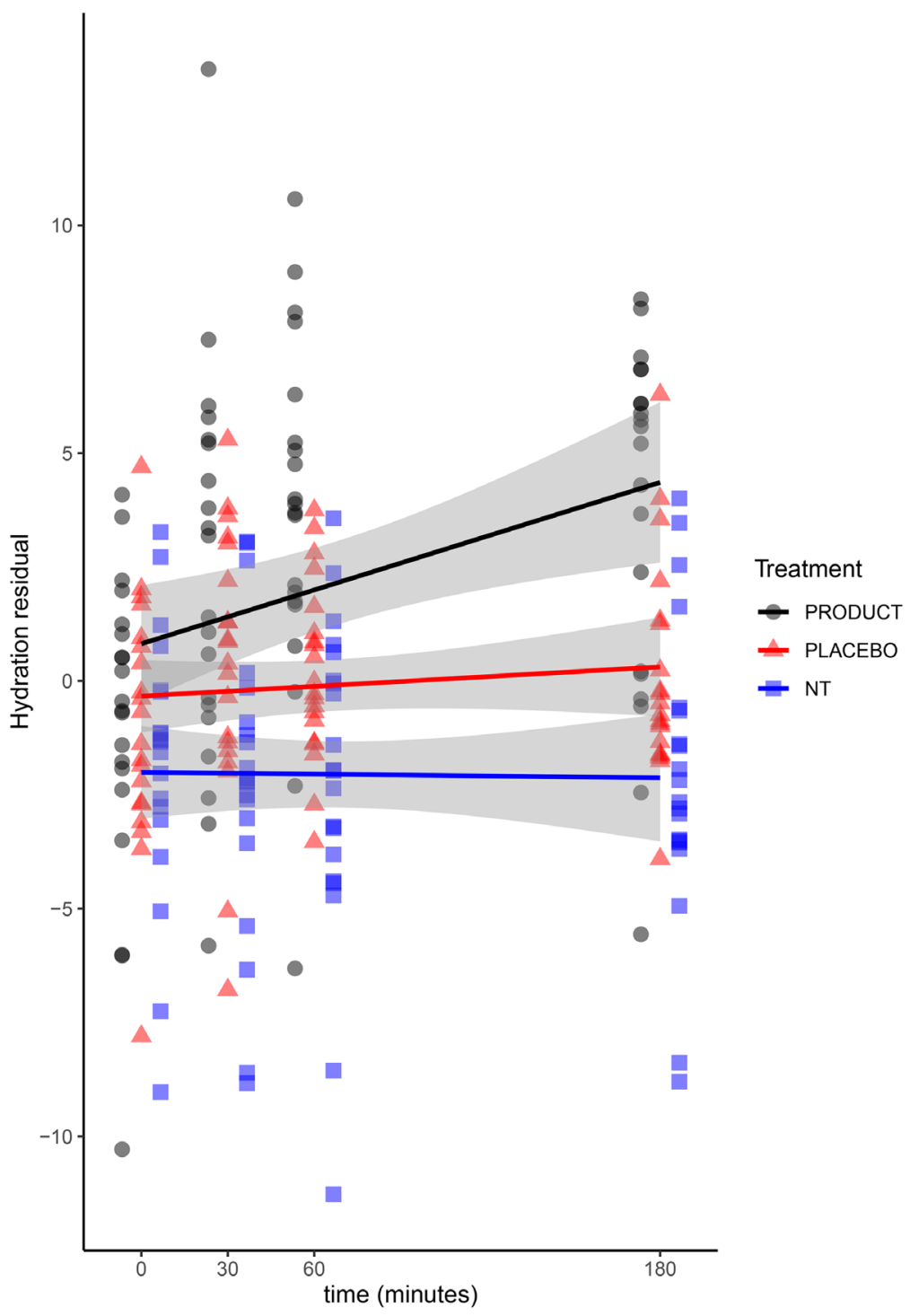

Figure 2. Skin hydration was assessed with a Corneometer after 1 application to the forearm area of either: Product: the tested cosmetic product; Placebo: carrier hydrogel (without peptides); NT: non-treated. Measurements were taken prior to application and were repeated for all groups on all participants at the indicated timepoints. 
Initial values for hydration were not significantly different between any of the three treatment groups (Figure 2). Hydration values for product-treated skin was $13.8 \%$ higher than that of NT skin at the first time point (30 minutes) ( $\mathrm{P}=$ 0.031 , pairwise t-test, $\mathrm{n}=20$ ). After 60 minutes, product-treated skin values were $17.5 \%$ higher than NT skin $(P=0.004$, pairwise $t$-test, $n=20)$ and $9.7 \%$ higher than placebo-treated skin ( $P=0.013$, pairwise t-test, $n=20)$. The hydrating effect was maintained until the 180-minute timepoint where product-treated skin hydration values were $17.5 \%$ higher than those for NT skin $(\mathrm{P}=0.003$, pairwise t-test, $\mathrm{n}=20)$ and $10.2 \%$ higher than placebo-treated skin $(\mathrm{P}=0.016$, pairwise $\mathrm{t}$-test, $\mathrm{n}=20$ ). At none of the observed points was there a significant difference between the placebo and NT skin hydration values.

Responses to the participant questionnaire were positive overall, with $90 \%$ rating the texture as either good or excellent, $80 \%$ rating the scent as either sufficient or good, $90 \%$ rating the comfort of the product as good or excellent. Eighty percent judged the product overall to be good or excellent and $95 \%$ of participants indicated that they would recommend the product to others. The responses are shown in Table 4 and reflect the opinions of all participants.

Table 3. ANOVA of hydration values.

\begin{tabular}{ccccc}
\hline \multirow{2}{*}{ Treatment } & \multicolumn{4}{c}{ ANOVA values } \\
\cline { 2 - 5 } & Difference & Lower & Upper & P.adj. \\
\hline Placebo - Product & -2.23725 & -3.525952 & -0.9485475 & 0001757 \\
NT- Product & -4.19575 & -5.484452 & -2.9070475 & 0.0000000 \\
NT - Placebno & -1.95850 & -3.247202 & -0.6697975 & 0.0012057 \\
\hline
\end{tabular}

An analysis of variance (ANOVA, Tukey multiple comparisons of means, 95\% family-wise confidence level) was also performed on treatment, using the model: Hydration $\sim$ Individual + Timepoint + Treatment + Timepoint: Treatment. The threshold for statistical significance was set at $5 \%(\mathrm{P}<0.05)$. P.adj: adjusted p-value.

Table 4. Questionnaire responses of the study participants.

\begin{tabular}{|c|c|c|c|c|c|c|}
\hline & \multirow{2}{*}{$n$} & \multicolumn{5}{|c|}{ Participant opinions (\%) } \\
\hline & & insufficient & poor & sufficient & good & excellent \\
\hline Texture of product & 20 & 0 & 0 & 10 & 60 & 30 \\
\hline Scent of product & 20 & 5 & 15 & 40 & 40 & 0 \\
\hline Comfort of product & 20 & 0 & 0 & 10 & 65 & 25 \\
\hline \multirow[t]{2}{*}{ General rating of product } & 20 & 0 & 0 & 20 & 65 & 15 \\
\hline & & Yes & No & & & \\
\hline $\begin{array}{c}\text { Would you recomment } \\
\text { the product? }\end{array}$ & 20 & 95 & 5 & & & \\
\hline
\end{tabular}




\section{Discussion}

The vulnerability of aged skin, in that it is thinner, less elastic, and more prone to injury represents an unmet clinical need, which will be exacerbated by an aging population and ever-increasing life expectancy. On the basis of published data, described in the introduction, which supports the effect of EGF, IGF, FGF, and IL-7 on different aspects of connective tissue and skin biology [15]-[20], [23] [24] [25] [29], we hypothesised that the inclusion of these peptide oligomers would increase the levels of connective tissue and ECM proteins in the skin. We proposed that this might manifest itself in numerous ways including, but not limited to, increased elasticity, volume, and firmness of the skin.

After 30 days of twice-daily use, we assessed the effect of a novel cosmetic product on selected parameters of skin physiology and observed the following significant results:

- $11.8 \%$ mean increase in elasticity

- $6.3 \%$ mean decrease in sagging

- $2.5 \%$ mean increase in luminosity

- Increase in mean brightness score from 0.9 to 1.9 (range $=0$ - 3)

Additionally, over a shorter timeframe, we observed an increase in skin hydration after application of the tested product with significantly greater hydration levels than NT or placebo-treated skin.

This combination of peptide isomers is responsible for the significant differences observed between the tested product and the placebo control in the hydration assay (Figure 2). This strongly suggests that the peptides within the tested product are capable of exerting their effects despite the absence of mechanical disruption of the SC. It is possible that the transdermal penetration of the peptides somehow facilitates increased transdermal co-penetration of hydrogel components, such as sodium hyaluronate, that increase the moisture content of the epidermis through the binding of a large number of water molecules, this might explain the duration of the moisturizing effect seen after application of the product. It is perhaps noteworthy that placebo application caused a significantly smaller increase in hydration than observed after application of the product, but one that lasted a similar duration, suggesting that similar mechanisms dictated the duration, but not the magnitude of the effect. This would be in line with an increased transdermal penetration of HA in the product-treated areas with respect to placebo-treated areas.

We propose that the known roles for the 4 peptides within the product [15] [16] [17] [18] [20] [23]-[28] in the process of wound healing [15] [17] [20] and soft tissue repair [23] are responsible for increased cell migration [28], increased cell turnover [16] and an upregulation in collagen [16] and elastin [21] [22] synthesis within the dermis which could explain the observed increase in skin elasticity and corresponding reduction in sagging of the skin.

The observed increase in elasticity represents an improvement over other peptide-containing formulations [33], and is similar to results obtained using oral 
supplements containing Lactobacillus [34], or topical formulations containing growth factors [35] or Pomegranate (Punica granatum) and Croton lechleri extracts [36], albeit over longer time-frames (12 and 6 weeks, respectively) than the current study. In terms of the capacity of the tested product to increase the moisture content of the skin, the effect was clear, but the short time-frame and the experimental design (analysis of hydration following a single application) makes comparison with other published studies difficult. However, despite the shorter duration, the results described herein are comparable with data from other studies over four [37] or six [36] weeks of use, and are greater than other reported results in including, oral probiotics used for four weeks [34] and application of topical $0.1 \%$ and $0.05 \%$ retinaldehyde over 3 months [38].

It would be interesting to see the effects of the same tested product on different ethnic groups. Testing hydration after longer periods would be useful to examine the duration of effects following one application. The effects of sustained use would also be of interest. These, and in vitro tests are warranted to demonstrate the ability of this product to affect collagen and elastin synthesis in the dermis which would provide a rational explanation for the observed effects on the skin in this study.

\section{Conclusion}

The potential of synthetic peptides to recapitulate the biological roles of endogenous molecules is a field of study that is gaining momentum. Here, in a 30-day clinical study of 20 participants, we provide evidence that topically applied peptides can affect rapid change in the physiological characteristics of the skin.

\section{Conflicts of Interest}

This study was sponsored by Ekuberg Pharma. Contrad Swiss SA owns and sells the product described in this manuscript. D. Carati is employed by Ekuberg Pharma. N. Fisher is employed by Contrad Swiss SA.

\section{References}

[1] Naval, J., Alonso, V. and Herranz, M.A. (2014) Genetic Polymorphisms and Skin Aging: The Identification of Population Genotypic Groups Holds Potential for Personalized Treatments. Clinical, Cosmetic and Investigational Dermatology, 7, 207-214. https://doi.org/10.2147/CCID.S55669

[2] Shekar, S.N., Luciano, M., Duffy, D.L. and Martin, N.G. (2005) Genetic and Environmental Influences on Skin Pattern Deterioration. Journal of Investigative Dermatology, 125, 1119-1129. https://doi.org/10.1111/j.0022-202X.2005.23961.x

[3] Fisher, G.J., et al. (2009) Collagen Fragmentation Promotes Oxidative Stress and Elevates Matrix Metalloproteinase-1 in Fibroblasts in Aged Human Skin. The American Journal of Pathology, 174, 101-114. https://doi.org/10.2353/ajpath.2009.080599

[4] Panich, U., Sittithumcharee, G., Rathviboon, N. and Jirawatnotai, S. (2016) Ultraviolet Radiation-Induced Skin Aging: The Role of DNA Damage and Oxidative Stress in Epidermal Stem Cell Damage Mediated Skin Aging. Stem Cells International, 2016, Article ID: 7370642. https://doi.org/10.1155/2016/7370642 
[5] Fisher, G.J., Wang, Z.Q., Datta, S.C., Varani, J., Kang, S. and Voorhees, J.J. (1997) Pathophysiology of Premature Skin Aging Induced by Ultraviolet Light. The New England Journal of Medicine, 337, 1419-1428. https://doi.org/10.1056/NEJM199711133372003

[6] Flament, F., Ye, C. and Amar, D. (2019) Assessing the Impact of an Aerial Chronic Urban Pollution (UP) on Some Facial Signs of Differently-Aged Chinese Men. International Journal of Cosmetic Science, 41, 450-461. https://doi.org/10.1111/ics.12558

[7] Boyd, A.S., Stasko, T., King, L.E., Cameron, G.S., Pearse, A.D. and Gaskell, S.A. (1999) Cigarette Smoking-Associated Elastotic Changes in the Skin. Journal of the American Academy of Dermatology, 41, 23-26. https://doi.org/10.1016/S0190-9622(99)70400-7

[8] Fisher, G.J., et al. (2002) Mechanisms of Photoaging and Chronological Skin Aging. Archives of Dermatology, 138, 1462-1470. https://doi.org/10.1001/archderm.138.11.1462

[9] Katayama, K., Armendariz-Borunda, J., Raghow, R., Kang, A.H. and Seyer, J.M. (1993) A Pentapeptide from Type I Procollagen Promotes Extracellular Matrix Production. Journal of Biological Chemistry, 268, 9941-9944.

[10] Park, H., An, E. and Cho Lee, A.-R. (2017) Effect of Palmitoyl-Pentapeptide (Pal-KTTKS) on Wound Contractile Process in Relation with Connective Tissue Growth Factor and $\alpha$-Smooth Muscle Actin Expression. Tissue Engineering and Regenerative Medicine, 14, 73-80. https://doi.org/10.1007/s13770-016-0017-y

[11] Tsai, W.-C., Hsu, C.-C., Chung, C.-Y., Lin, M.-S., Li, S.-L. and Pang, J.-H.S. (2007) The Pentapeptide KTTKS Promoting the Expressions of Type I Collagen and Transforming Growth Factor-Beta of Tendon Cells. Journal of Orthopaedic Research, 25, 1629-1634. https://doi.org/10.1002/jor.20455

[12] Aguilar-Toalá, J.E., Hernández-Mendoza, A., González-Córdova, A.F., Vallejo-Cordoba, B. and Liceaga, A.M. (2019) Potential Role of Natural Bioactive Peptides for Development of Cosmeceutical Skin Products. Peptides, 122, Article ID: 170170. https://doi.org/10.1016/j.peptides.2019.170170

[13] Rodrigues-Barata, A.R. and Camacho-Martínez, F.M. (2013) Undesirable Effects after Treatment with Dermal Fillers. Journal of Drugs in Dermatology, 12, e59-e62.

[14] Schwarz, A., et al. (2001) Interleukin-12 Suppresses Ultraviolet Radiation-Induced Apoptosis by Inducing DNA Repair. Nature Cell Biology, 4, 26-31.

https://doi.org/10.1038/ncb717

[15] Franzén, L., Ghassemifar, R. and Schultz, G. (1993) Specific Binding of EGF in Connective Tissue Repair. European Journal of Cell Biology, 60, 346-350.

[16] Amara, H.B., Thoma, D.S., Schwarz, F., Song, H.Y., Capetillo, J. and Koo, K.-T. (2019) Healing Kinetics of Oral Soft Tissue Wounds Treated with Recombinant Epidermal Growth Factor: Translation from a Canine Model. Journal of Clinical Periodontology, 46, 105-117. https://doi.org/10.1111/jcpe.13035

[17] Esquirol-Caussa, J. and Herrero-Vila, E. (2019) Human Recombinant Epidermal Growth Factor in Skin Lesions: 77 Cases in EPItelizando Project. Journal of Dermatological Treatment, 30, 96-101. https://doi.org/10.1080/09546634.2018.1468546

[18] Lewis, D.A., Travers, J.B., Somani, A.-K. and Spandau, D.F. (2010) The IGF-1/IGF-1R Signaling Axis in the Skin: A New Role for the Dermis in Aging-Associated Skin Cancer. Oncogene, 29, 1475-1485. https://doi.org/10.1038/onc.2009.440

[19] Lewis, D.A., Yi, Q., Travers, J.B. and Spandau, D.F. (2008) UVB-Induced Senescence in Human Keratinocytes Requires a Functional Insulin-Like Growth Factor-1 
Receptor and p53. Molecular Biology of the Cell, 19, 1346-1353. https://doi.org/10.1091/mbc.e07-10-1041

[20] Gong, F., et al. (2019) Effect of Insulin-Like Growth Factor-1 on Promoting Healing of Skin Ulcers in Diabetic Rats. Journal of Biological Regulators and Homeostatic Agents, 33, 687-694.

[21] Wolfe, B.L., et al. (1993) Insulin-Like Growth Factor-I Regulates Transcription of the Elastin Gene. Journal of Biological Chemistry, 268, 12418-12426.

[22] Qa'aty, N., Vincent, M., Wang, Y., Wang, A., Mitts, T.F. and Hinek, A. (2015) Synthetic Ligands of the Elastin Receptor Induce Elastogenesis in Human Dermal Fibroblasts via Activation of Their IGF-1 Receptors. Journal of Dermatological Science, 80, 175-185. https://doi.org/10.1016/j.jdermsci.2015.10.001

[23] Kakudo, N., Morimoto, N., Ogawa, T. and Kusumoto, K. (2019) Effects of Fibroblast Growth Factor-2 Combined with a Collagen/Gelatin Sponge for Adipogenesis in the Mouse Subcutis. Annals of Plastic Surgery, 84, 216-221. https://doi.org/10.1097/SAP.0000000000002046

[24] Li, Z., et al. (2018) Sustained Release of Basic Fibroblast Growth Factor Using Gelatin Hydrogel Improved Left Ventricular Function through the Alteration of Collagen Subtype in a Rat Chronic Myocardial Infarction Model. General Thoracic and Cardiovascular Surgery, 66, 641-647. https://doi.org/10.1007/s11748-018-0969-z

[25] Fujihara, C., et al. (2019) Fibroblast Growth Factor-2 Inhibits CD40-Mediated Periodontal Inflammation. Journal of Cellular Physiology, 234, 7149-7160. https://doi.org/10.1002/jcp.27469

[26] Roye, O., et al. (1998) Dermal Endothelial Cells and Keratinocytes Produce IL-7 in Vivo after Human Schistosoma mansoni Percutaneous Infection. The Journal of Immunology, 161, 4161-4168.

[27] Heufler, C., et al. (1993) Interleukin 7 Is Produced by Murine and Human Keratinocytes. Journal of Experimental Medicine, 178, 1109-1114. https://doi.org/10.1084/jem.178.3.1109

[28] Bartlett, A., Sanders, A.J., Ruge, F., Harding, K.G. and Jiang, W.G. (2016) Potential Implications of Interleukin-7 in Chronic Wound Healing. Experimental and Therapeutic Medicine, 12, 33-40. https://doi.org/10.3892/etm.2016.3263

[29] Barata, J.T., Durum, S.K. and Seddon, B. (2019) Flip the Coin: IL-7 and IL-7R in Health and Disease. Nature Immunology, 20, 1584-1593. https://doi.org/10.1038/s41590-019-0479-x

[30] Phetcharat, L., Wongsuphasawat, K. and Winther, K. (2015) The Effectiveness of a Standardized Rose Hip Powder, Containing Seeds and Shells of Rosa canina, on Cell Longevity, Skin Wrinkles, Moisture, and Elasticity. Clinical Interventions in Aging, 10, 1849-1856. https://doi.org/10.2147/CIA.S90092

[31] Couteau, C., Coiffard, L.J.M. and Sébille-Rivain, V. (2006) Influence of Excipients on Moisturizing Effect of Urea. Drug Development and Industrial Pharmacy, 32, 239-242. https://doi.org/10.1080/03639040500466361

[32] 3.6.1 R Core Team. R: A Language and Environment for Statistical Computing. R Foundation for Statistical Computing, Vienna. https://www.R-project.org

[33] Hahn, H.J., et al. (2016) Instrumental Evaluation of Anti-Aging Effects of Cosmetic Formulations Containing Palmitoyl Peptides, Silybum marianum Seed Oil, Vitamin E and Other Functional Ingredients on Aged Human Skin. Experimental and Therapeutic Medicine, 12, 1171-1176. https://doi.org/10.3892/etm.2016.3447

[34] Lee, D.E., et al. (2015) Clinical Evidence of Effects of Lactobacillus plantarum 
HY7714 on Skin Aging: A Randomized, Double Blind, Placebo-Controlled Study. Journal of Microbiology and Biotechnology, 25, 2160-2168. https://doi.org/10.4014/jmb.1509.09021

[35] Barone, F., Bashey, S. and Woodin Jr., F.W. (2019) Clinical Evidence of Dermal and Epidermal Restructuring from a Biologically Active Growth Factor Serum for Skin Rejuvenation. Journal of Drugs in Dermatology, 18, 290-295.

[36] Bogdan, C., Iurian, S., Tomuta, I. and Moldovan, M. (2017) Improvement of Skin Condition in Striae Distensae: Development, Characterization and Clinical Efficacy of a Cosmetic Product Containing Punica granatum Seed Oil and Croton lechleri Resin Extract. Drug Design, Development and Therapy, 11, 521-531. https://doi.org/10.2147/DDDT.S128470

[37] Draelos, Z.D., Levy, S.B., Lutrario, C. and Gunt, H. (2018) Evaluation of the Performance of a Nature-Based Sensitive Skin Regimen in Subjects with Clinically Diagnosed Sensitive Skin. Journal of Drugs in Dermatology, 17, 908-913.

[38] Kwon, H.S., Lee, J.H., Kim, G.M. and Bae, J.M. (2018) Efficacy and Safety of Retinaldehyde $0.1 \%$ and $0.05 \%$ Creams Used to Treat Photoaged Skin: A Randomized Double-Blind Controlled Trial. Journal of Cosmetic Dermatology, 17, 471-476. https://doi.org/10.1111/jocd.12551 